\title{
Osthole induces apoptosis of the HT-29 cells via endoplasmic reticulum stress and autophagy
}

\author{
XING-HUA ZHOU $^{1}$, JIAN KANG ${ }^{1}$, ZHEN-DONG ZHONG ${ }^{2}$ and YUE CHENG ${ }^{1}$ \\ ${ }^{1}$ Department of Anorectal Diseases, Hospital of Chengdu University of Traditional \\ Chinese Medicine, Chengdu, Sichuan 610075; ${ }^{2}$ Animal Experiment Research Institute, \\ Sichuan Provincial People's Hospital, Chengdu, Sichuan 610000, P.R. China
}

Received January 23, 2021; Accepted June 18, 2021

DOI: $10.3892 / \mathrm{ol} .2021 .12987$

\begin{abstract}
Endoplasmic reticulum stress (ERS) and autophagy are important pathways, which induce apoptosis of tumor cells. Osthole has been demonstrated to exert anticancer effects via the induction of apoptosis in several human colon cancer lines, but the mechanism underlying its involvement in the induction of ERS and autophagy in the human HT-29 colorectal cancer cell line remains unknown. The present study aimed to identify the possible signaling pathways involved in osthole-induced apoptosis of HT29 cells. Methodologically, colony formation and Cell Counting Kit-8 assays were used to assess cell proliferation and viability, respectively, while flow cytometry was performed to investigate apoptosis. Signaling pathways, including apoptosis, autophagy and ERS, were also investigated in the HT-29 cell line using western blot analysis. The results demonstrated that osthole inhibited cellular proliferation and viability in a dose-dependent manner. In addition, osthole induced the expression level of proteins associated with mitochondria-mediated cell apoptosis, autophagy and ERS. The association between autophagy and ERS in osthole-induced apoptosis in the HT-29 cell line was further clarified. Inhibiting cell autophagy with the inhibitor, 3-methyladenine, suppressed osthole-induced cell apoptosis and enhanced osthole-induced ERS. By contrast, alleviating ERS with the inhibitor, 4-phenylbutyric acid attenuated osthole-induced cell apoptosis and autophagy. In conclusion, osthole could significantly suppress the proliferation and viability of the HT-29 colorectal cancer cell line and induce cell apoptosis via autophagy and ERS. Furthermore, ERS may play a more important role in osthole-induced cell apoptosis.
\end{abstract}

Correspondence to: Dr Yue Cheng, Department of Anorectal Diseases, Hospital of Chengdu University of Traditional Chinese Medicine, 39 Shi'er Qiao Road, Jinniu, Chengdu, Sichuan 610075, P.R. China

E-mail: msmscycy@hotmail.com

Key words: osthole, apoptosis, endoplasmic reticulum stress, autophagy, colon cancer

\section{Introduction}

Colon cancer is one of the most commonly diagnosed types of cancer worldwide and was ranked 4th among the most commonly diagnosed cancers in 2018 (1). Thus, colon cancer represents a major global public health concern. Conventional anticancer therapies, including surgery and chemotherapy, remain the most effective strategies for treatment; however, drug resistance develops in the majority of patients receiving chemotherapy (2). Colon cancer has poor clinical outcomes. Therefore, there is an urgent requirement to identify alternative anticancer agents to improve future treatment.

Osthole is a natural coumarin-derivative and bioactive compound extracted from the fruit of Cnidium monnieri (3). Its chemical formula is $\mathrm{C}_{15} \mathrm{H}_{16} \mathrm{O}_{3}$. It has been reported that osthole exhibited a broad range of pharmacological activities, including anti-osteoporotic, anti-inflammatory, cardiovascular and neuroprotective properties (4-7), as well as having anticancer effects, which have been demonstrated in certain types of cancer cells, such as breast, ovarian and lung cancer cells $(3,8,9)$. In addition, osthole induced cell death in human HCT116 and SW480 colon cancer cell lines (10). Osthole exerts anticancer effects by inhibiting cell proliferation and invasion, which may be associated with the induction of apoptosis and cell cycle arrest (11). However, the target of osthole-induced apoptosis of human HT-29 colorectal cancer cell line remains unclear.

Substantial efforts have been made to determine the molecular mechanisms that underlie cancer development and progression. Apoptosis and autophagy are 2 types of programmed cell death (12). Autophagy is induced in response to various stresses that ultimately lead to apoptosis and remove unnecessary or dysfunctional cytoplasmic components; therefore, autophagy plays an important role in various cellular functions, such as proliferation, apoptosis and epithelial-mesenchymal transition (8). The endoplasmic reticulum (ER) is the foremost intracellular compartment of the secretory pathway in eukaryotic cells (13). Disruption of ER homeostasis causes the accumulation of misfolded/unfolded proteins in the ER lumen, which contributes to ER stress (ERS). The unfolded protein response (UPR) is activated in response to increased ERS, and orchestrates the recovery of homeostasis or triggers apoptosis, depending on the 
degree and duration of damage or stress (14-16). The UPR is governed by the action of 3 signaling proteins/transmembrane ERS sensors, namely inositol-requiring enzyme $1 \alpha$ (IRE1 $\alpha$ ), protein kinase R (PKR)-like ER kinase (PERK) and activating transcription factor 6 (ATF6) (17). Persistent and severe ERS can switch the cytoprotective functions of UPR and autophagy into cell death programs (18).

As a potential anticancer agent, the effects of osthole on the apoptosis of colorectal cancer cells and the underlying mechanisms are poorly understood. The present study aimed to investigate the effects of osthole treatment on HT-29 cells with respect to its possible role in ERS, autophagy and apoptosis.

\section{Materials and methods}

Cell culture and treatments. The human HT-29 colorectal cancer cell line was purchased from Procell Life Science \& Technology Co., Ltd. (cat. no. CL-0118), and authenticated using STR profiling. The cells were cultured in Dulbeccos modified Eagle's medium (DMEM), supplemented with $10 \%$ heat-inactivated fetal bovine serum, $100 \mathrm{U} / \mathrm{ml}$ penicillin and $100 \mu \mathrm{g} / \mathrm{ml}$ streptomycin (all from Gibco; Thermo Fisher Scientific, Inc.). Then, the cells were maintained at $37^{\circ} \mathrm{C}$ in a humidified atmosphere with $5 \% \mathrm{CO}_{2}$.

Osthole was purchased from Shanghai Aladdin Biochemical Technology Co., Ltd., (cat. no. O101698) and dissolved in DMSO (Sigma-Aldrich; Merck KGaA), then diluted in DMEM to the desired final concentration (100, 50 and $25 \mu \mathrm{M})$. The HT-29 cell line was randomly divided into 4 different treatment groups: i) HT-29 cells were treated with $0.1 \%$ DMSO as the control and 100, 50 and $25 \mu \mathrm{M}$ osthole for $24 \mathrm{~h}$ separately. All subsequent experiments were performed using $50 \mu \mathrm{M}$ osthole. ii) HT-29 cells were treated with 10 mM 3-methyladenine (3MA; Sigma-Aldrich; Merck $\mathrm{KGaA}$ ), osthole and 3MA + osthole for $24 \mathrm{~h}$ separately, with $0.1 \%$ DMSO as the control. iii) HT-29 cells were treated with 10 mM 4-phenylbutyric acid (4-PBA; Sigma-Aldrich; Merck $\mathrm{KGaA}$ ), osthole and 4-PBA + osthole for $24 \mathrm{~h}$ separately, with $0.1 \%$ DMSO as the control. iv) HT-29 cells were treated with osthole, 3MA, 3MA + osthole, 4-PBA, 4-PBA + osthole and $3 \mathrm{MA}+$ osthole + 4-PBA for 24 h separately, with $0.1 \%$ DMSO as the control. The concentrations of 3MA, 4-PBA and osthole were referenced to the doses most commonly used in the other experimental studies (19-21).

Colony formation assay. The effects of osthole on the proliferation of the HT-29 cell line was measured using colony formation analysis. The HT-29 cells, treated as aforementioned, were seeded into 6-well culture plates separately, then cultured under normal conditions for 7 days to form colonies. Subsequently, the colonies were fixed with $4 \%$ paraformaldehyde (Sigma-Aldrich; Merck KGaA) for $30 \mathrm{~min}$ and stained with $0.1 \%$ crystal violet (Sigma-Aldrich; Merck KGaA) for $20 \mathrm{~min}$ at room temperature. Images were captured with a digital camera and colonies $\sim 0.3-1.0 \mathrm{~mm}$ in size were counted.

Cell viability assay. The HT-29 cells were seeded into 96-well culture plates, at a density of $5 \times 10^{3}$ per well. Following treatment as aforementioned for the first 3 groups, cell viability was detected using a Cell Counting Kit-8 (CCK-8; Beyotime Institute of Biotechnology), according to the manufacturer's instructions; $10 \mu \mathrm{l} \mathrm{CCK}-8$ solution was added and incubated for $1 \mathrm{~h}$ at $37^{\circ} \mathrm{C}$. The absorbance was measured at $450 \mathrm{~nm}$ using a microplate spectrophotometer (cat. no. 1681150; Bio-Rad Laboratories, Inc.) and cell viability was calculated using the following formula: Cell viability $(\%)=\left(\mathrm{A}_{\text {experimental }}-\mathrm{A}_{\text {blank }}\right) /\left(\mathrm{A}_{\text {control }}-\mathrm{A}_{\text {blank }}\right) \times 100 \%$.

Flow cytometry. The effects of osthole on the early and late apoptosis of HT-29 cells was determined by flow cytometry. Following all four different treatments, the HT-29 cells were harvested, washed twice with PBS (Sigma-Aldrich; Merck $\mathrm{KGaA}$ ), centrifuged at $300 \mathrm{x} \mathrm{g}$ for $5 \mathrm{~min}$ at room temperature. The next operation was performed using the apoptosis kit (Nanjing KeyGen Biotech Co., Ltd.); the cells were gently resuspended in binding buffer $(500 \mu \mathrm{l})$ and incubated with Annexin V-APC (5 $\mu \mathrm{l})$ and PI $(5 \mu \mathrm{l})$ in the dark for $15 \mathrm{~min}$. The cells were analyzed using a FACSCalibur ${ }^{\mathrm{TM}}$ flow cytometer (BD Biosciences) and FlowJo software version 10.6.2 (Tree Star, Inc.).

Western blot analysis. Following different treatments, the HT-29 cells were collected. Total cellular protein was obtained by lysing the cells with RIPA buffer (cat. no. R0020) and the protein concentration was performed using a BCA Protein Assay kit (cat. no. PC0020) (both from Beijing Solarbio Science \& Technology Co., Ltd.). The protein samples were denatured, and $50 \mu$ g total protein samples were separated using $10 \%$ SDS-PAGE, and subsequently electroblotted onto PVDF membranes (EMD Millipore). The membranes were blocked for $2 \mathrm{~h}$ at room temperature with TBS and $0.05 \%$ Tween-20 (TBST), containing $5 \%$ skimmed milk, then the membranes were incubated overnight at $4^{\circ} \mathrm{C}$ with anti- $\beta$-actin (1:1,000; cat. no. ab8227; Abcam), anti-Bax (1:1,000; cat. no. ab53154; Abcam), anti-Bcl-2 (1:1,000; cat. no. ab59348; Abcam), anti-cleaved caspase-3 (1:1,000; cat. no. ab2302; Abcam), anti-p62 (1:500; cat. no. ab155686; Abcam), anti-microtubule-associated protein light chain 3 (LC3; 1:1,000; cat. no. 12741; Cell Signaling Technology, Inc.), anti-CHOP (1:1,000; cat. no. 2895; Cell Signaling Technology, Inc.), anti-PERK (1:1,000; cat. no. 5683; Cell Signaling Technology, Inc.), anti-phosphorylated (p)-PERK (1:1,000; cat. no. 3179; Cell Signaling Technology, Inc.), anti-eukaryotic initiation factor 2 (eIF2) $\alpha$ (1:1,000; cat. no. 5324; Cell Signaling Technology, Inc.), anti-p-eIF2 $\alpha$ (1:1,000; cat. no. 3398; Cell Signaling Technology, Inc.) and anti-78 kDa glucose-regulated protein (GRP78; 1:1,000; ProteinTech Group, Inc.) antibodies. After washing 3 times with TBST, the membranes were incubated with a HRP-conjugated secondary antibody $(1: 2,000$; cat. no. sc-2748; Santa Cruz Biotechnology, Inc.) for $2 \mathrm{~h}$ at room temperature. The proteins on the membranes were visualized with an enhanced chemiluminescence detection kit (Bio-Rad Laboratories, Inc.) using a ChemiScope 6000 imaging system (Clinx Science Instruments Co., Ltd.). Densitometry was performed using ImageJ software version 1.8.0 (National Institutes of Health).

Statistical analysis. All experiments were independently repeated at least 3 times and all statistical analyses were performed using SPSS software version 20.0 (IBM Corp.). All the data are presented as the mean \pm SD. Statistical comparisons 
A

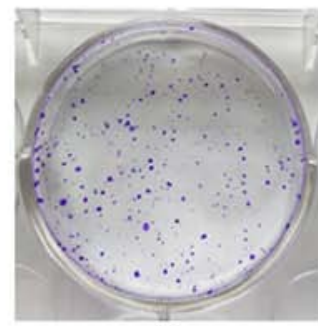

0

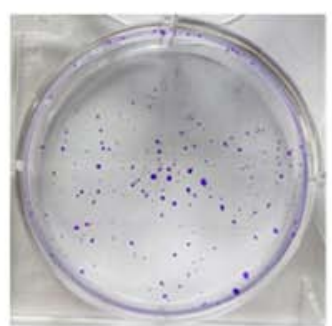

100

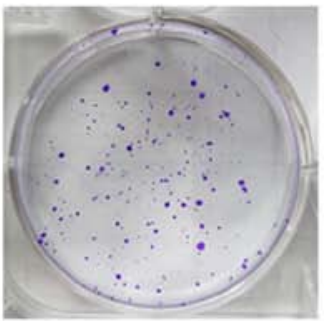

50

Osthole $(\mu \mathrm{M})$
B

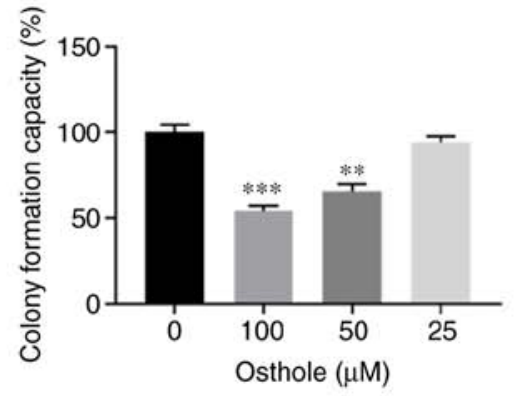

C

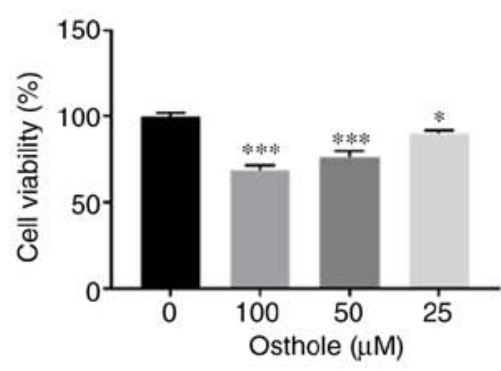

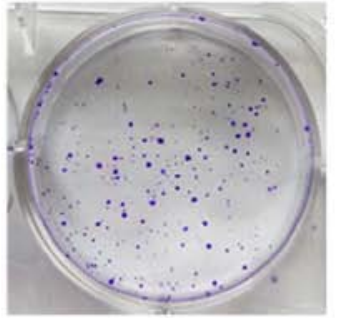

25
D

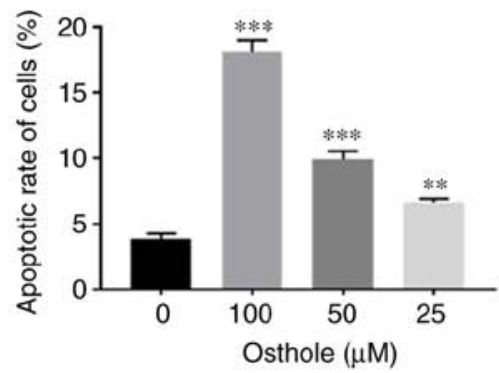

E Osthole $(\mu \mathrm{M})$
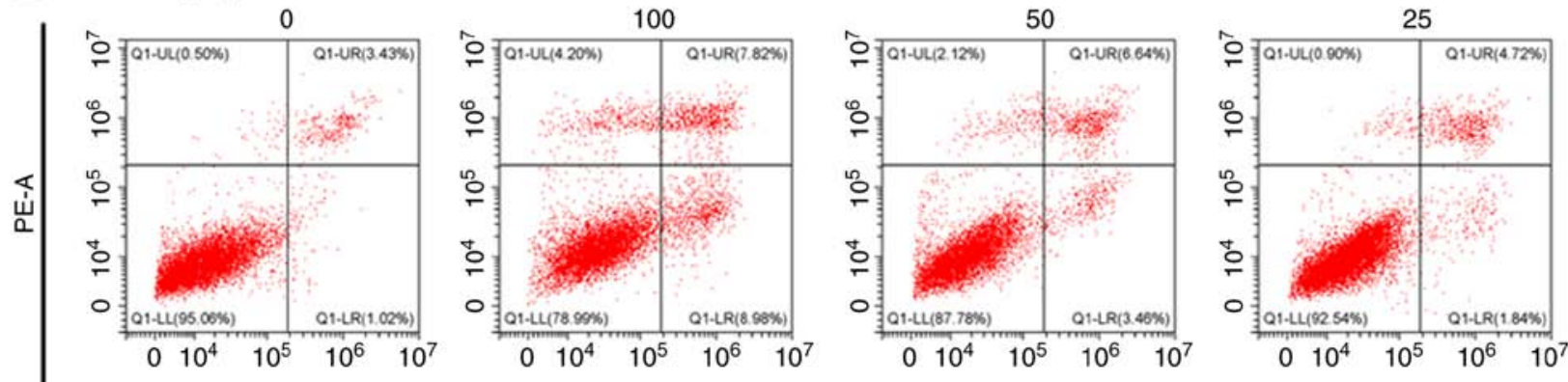

APC-A

$\mathrm{F}$

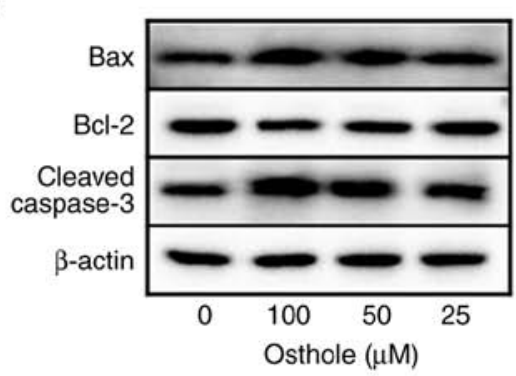

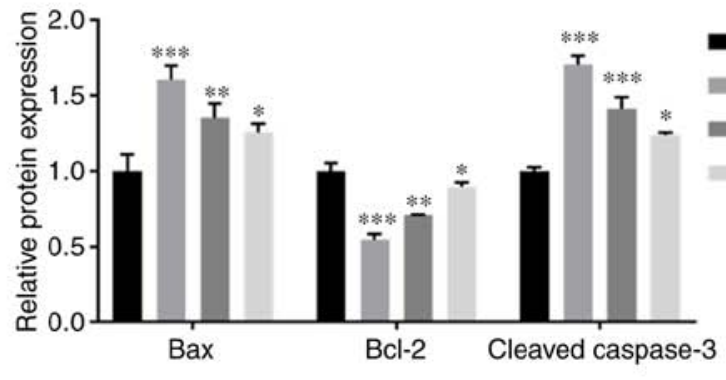

Figure 1. Osthole inhibits proliferation and viability, and induces apoptosis of the HT-29 cell line. The proliferation and viability of the HT-29 cells treated with osthole $(0,100,50$ and $25 \mu \mathrm{M})$ for $24 \mathrm{~h}$ was detected using (A and B) colony formation and (C) Cell Counting Kit-8 assays, respectively. (D and E) HT-29 cell apoptosis was detected using flow cytometry. (F) The expression levels of the apoptosis-associated proteins Bax, Bcl-2 and cleaved caspase-3 were determined using western blot analysis, with $\beta$-actin as a loading control. ${ }^{*} \mathrm{P}<0.05,{ }^{* *} \mathrm{P}<0.01,{ }^{* * * *} \mathrm{P}<0.001$ vs. control group $(0 \mu \mathrm{M})$.

were performed using one-way ANOVA and Tukey's post hoc test for differences among multiple groups and an unpaired $\mathrm{t}$-test for differences between 2 groups. $\mathrm{P}<0.05$ was considered to indicate a statistically significant difference.

\section{Results}

Osthole effectively suppresses proliferation and induces apoptosis in the HT-29 cell line. Osthole has been reported to exert inhibitory effects on several human cancer cells $(3,8-9)$, and to have anticancer effects on human HCT116 and SW480 colon cancer cells (10). To investigate whether osthole has a similar inhibitory role in HT-29 colorectal cells, proliferation and apoptosis were investigated. The results of the colony formation assay revealed that osthole, at $50 \mu \mathrm{M}$, exerted a significant inhibitory effect on the proliferation of the HT-29 cells and there was also a dose-dependent inhibitory effect $(\mathrm{P}<0.001)$ (Fig. 1A and B). Similarly, the CCK-8 assay also 
A

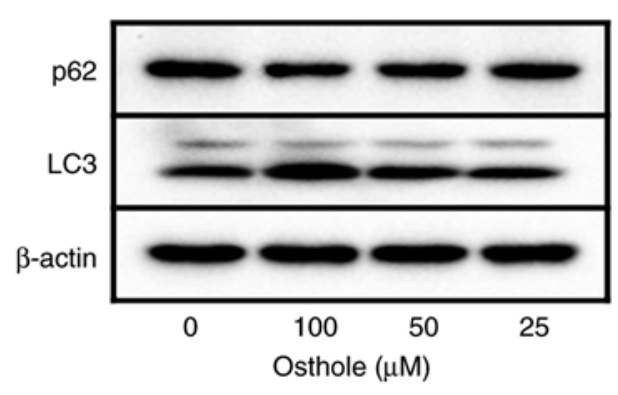

B

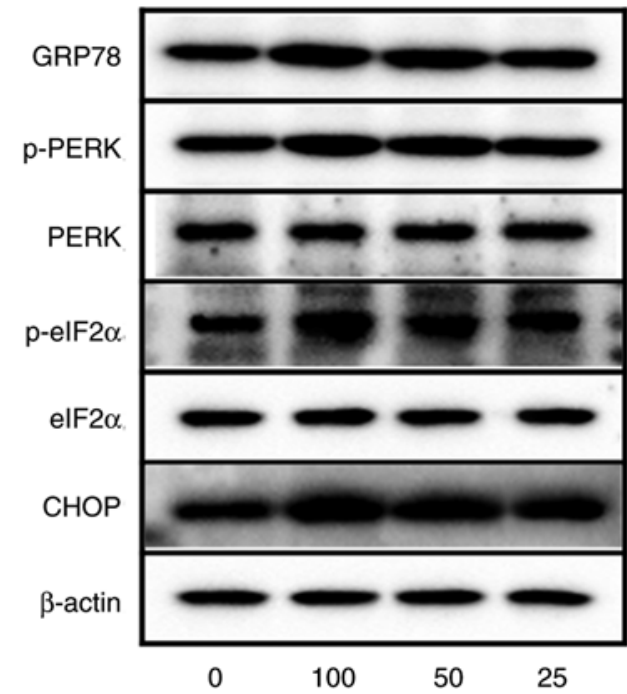

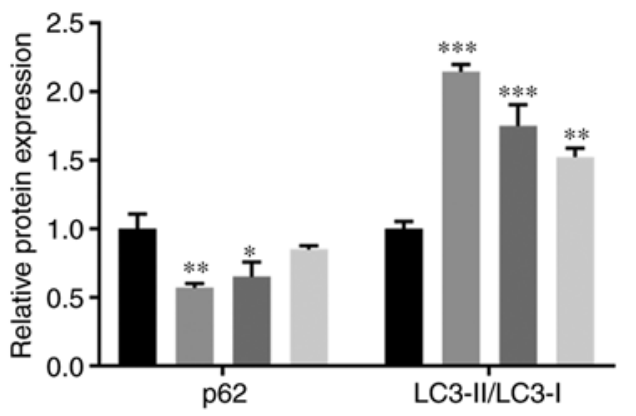

$0 \mu \mathrm{M}$ Osthole

$100 \mu \mathrm{M}$ Osthole

$50 \mu \mathrm{M}$ Osthole

$25 \mu \mathrm{M}$ Osthole

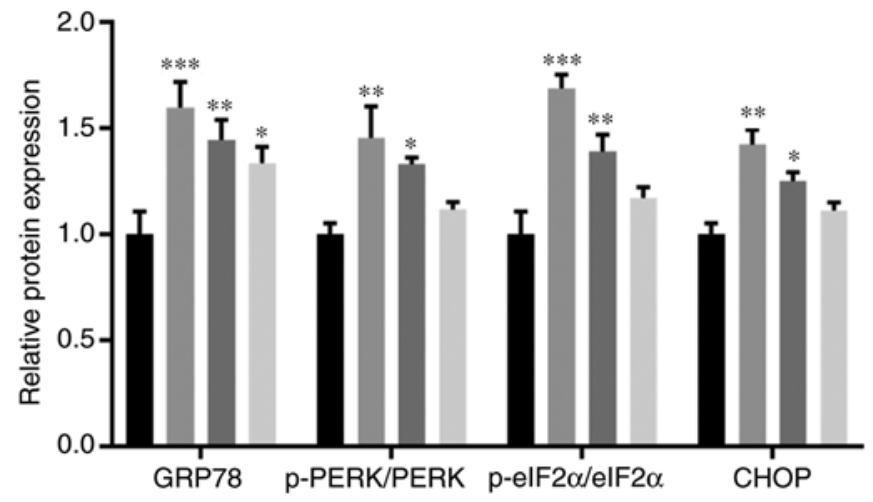

Figure 2. Effects of osthole on the protein expression levels of autophagy- and endoplasmic reticulum stress-related markers in the HT-29 cell line. The protein expression levels of (A) p62 and LC3-II/LC3-I, (B) GRP78, p-PERK/PERK, p-elF2 $\alpha /$ elF2 $\alpha$ and CHOP were determined using western blot analysis. $\beta$-actin was used as a loading control. ${ }^{*} \mathrm{P}<0.05,{ }^{* *} \mathrm{P}<0.01,{ }^{* * * *} \mathrm{P}<0.001$ vs. control group $(0 \mu \mathrm{M})$. LC3, microtubule-associated protein light chain 3; GRP78, $78 \mathrm{kDa}$ glucose-regulated protein; p-, phosphorylated; PERK, protein kinase R (PKR)-like endoplasmic reticulum kinase; elF2 $\alpha$, eukaryotic initiation factor $2 \alpha$.

showed that osthole significantly inhibited cell viability in a dose-dependent manner ( $\mathrm{P}<0.05$; Fig. 1C).

As osthole treatment reduced the viability of the HT-29 cells, it was subsequently investigated whether osthole could induced apoptosis. The results of flow cytometry revealed that the apoptotic rate of the HT-29 cell line was significantly increased, in a dose-dependent manner $(\mathrm{P}<0.01)$ (Fig. 1D and E). This finding was further confirmed following detection of the expression level of apoptosis-related proteins using western blot analysis. It was demonstrated that the protein expression levels of Bax and cleaved caspase- 3 were significantly increased, whereas the protein expression level of Bcl-2 was significantly decreased, in a dose-dependent manner $(\mathrm{P}<0.05$; Fig. 1F). These results suggested that osthole inhibited the proliferation and induced apoptosis in the HT-29 cell line.

Osthole activates autophagy and ERS in the HT-29 cell line. Autophagy and ERS play important roles in cell apoptosis $(22,23)$; therefore, the effects of osthole on autophagy and ERS were detected using western blot analysis. The results demonstrated that the protein expression level of p62 was significantly decreased and the protein expression level of LC3-II/LC3-I was significantly increased following osthole treatment, in a dose-dependent manner $(\mathrm{P}<0.05$; Fig. 2A). In addition, the protein expression level of GRP78 was significantly increased following treatment with $25 \mu \mathrm{M}$ osthole $(\mathrm{P}<0.05)$, and the protein expression levels of $\mathrm{p}$-PERK/PERK, p-elF $2 \alpha /$ elF $2 \alpha$ and CHOP were significantly increased following treatment with $50 \mu \mathrm{M}$ osthole ( $\mathrm{P}<0.05$; Fig. $2 \mathrm{~B}$ ). These results showed that osthole induced autophagy and ERS in the HT-29 cell line.

Effects of autophagy on osthole-induced HT-29 cell apoptosis. To further determine the role of autophagy in osthole-induced HT-29 cell apoptosis, the HT-29 cell line was treated with osthole and the autophagy inhibitor, 3MA, and cellular proliferation and viability was examined using colony formation and CCK-8 assays, respectively. As shown in Fig. 3A and B, compared with control group, osthole inhibited cell proliferation $(\mathrm{P}<0.05)$, whereas 3MA increased the proliferation of HT-29 cells, but not significantly ( $\mathrm{P}>0.05)$. Notably, co-treatment with osthole and 3MA significantly inhibited cell proliferation compared with that in the 3MA group $(\mathrm{P}<0.05)$; however, there were no significant differences compared with that in the osthole group ( $\mathrm{P}>0.05)$. Similarly, the CCK-8 assay also revealed that osthole significantly inhibited cell viability compared with that in the control group $(\mathrm{P}<0.001)$, and there was a significant decrease in cell viability between the $3 \mathrm{MA}$ and 3MA + osthole groups $(\mathrm{P}<0.001)$. However, co-treatment with osthole and 3MA did not significantly inhibit cell viability compared with that in the osthole group ( $\mathrm{P}>0.05$; Fig. $3 \mathrm{C}$ ). 
A

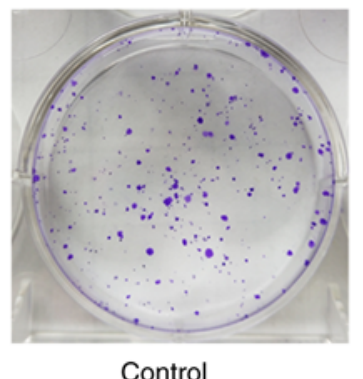

B

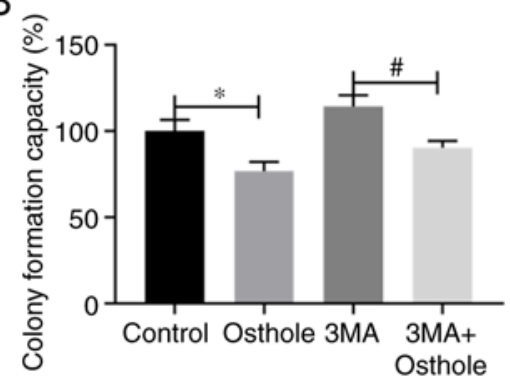

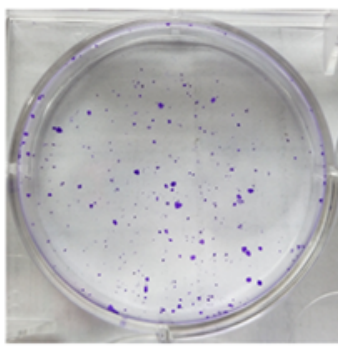

Osthole

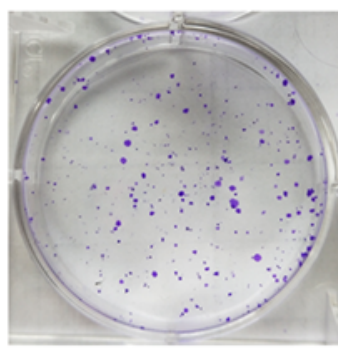

3MA

C

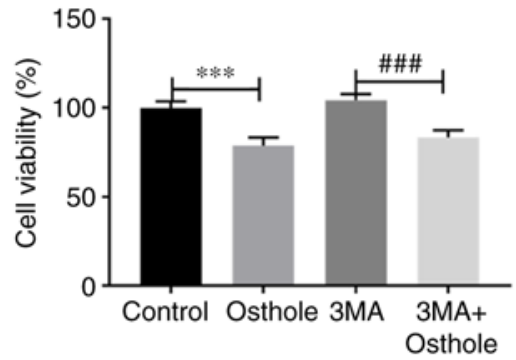

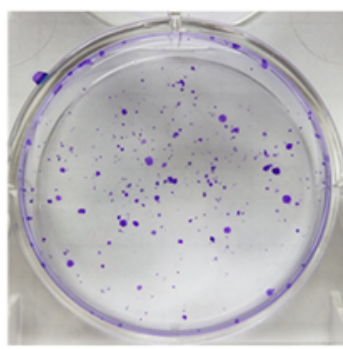

$3 \mathrm{MA}+$ Osthole
D

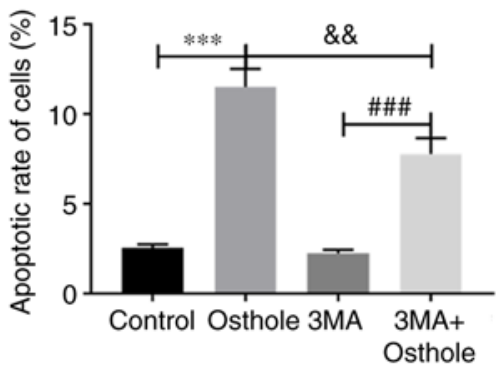

E

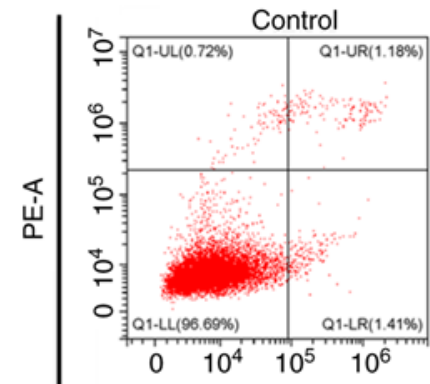

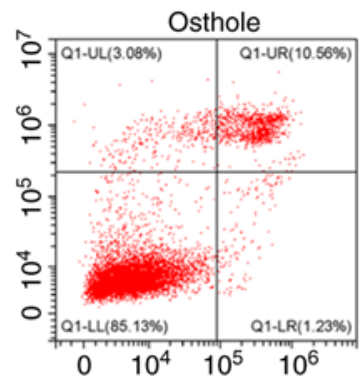

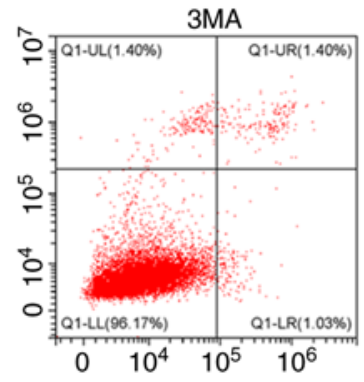

F

APC-A
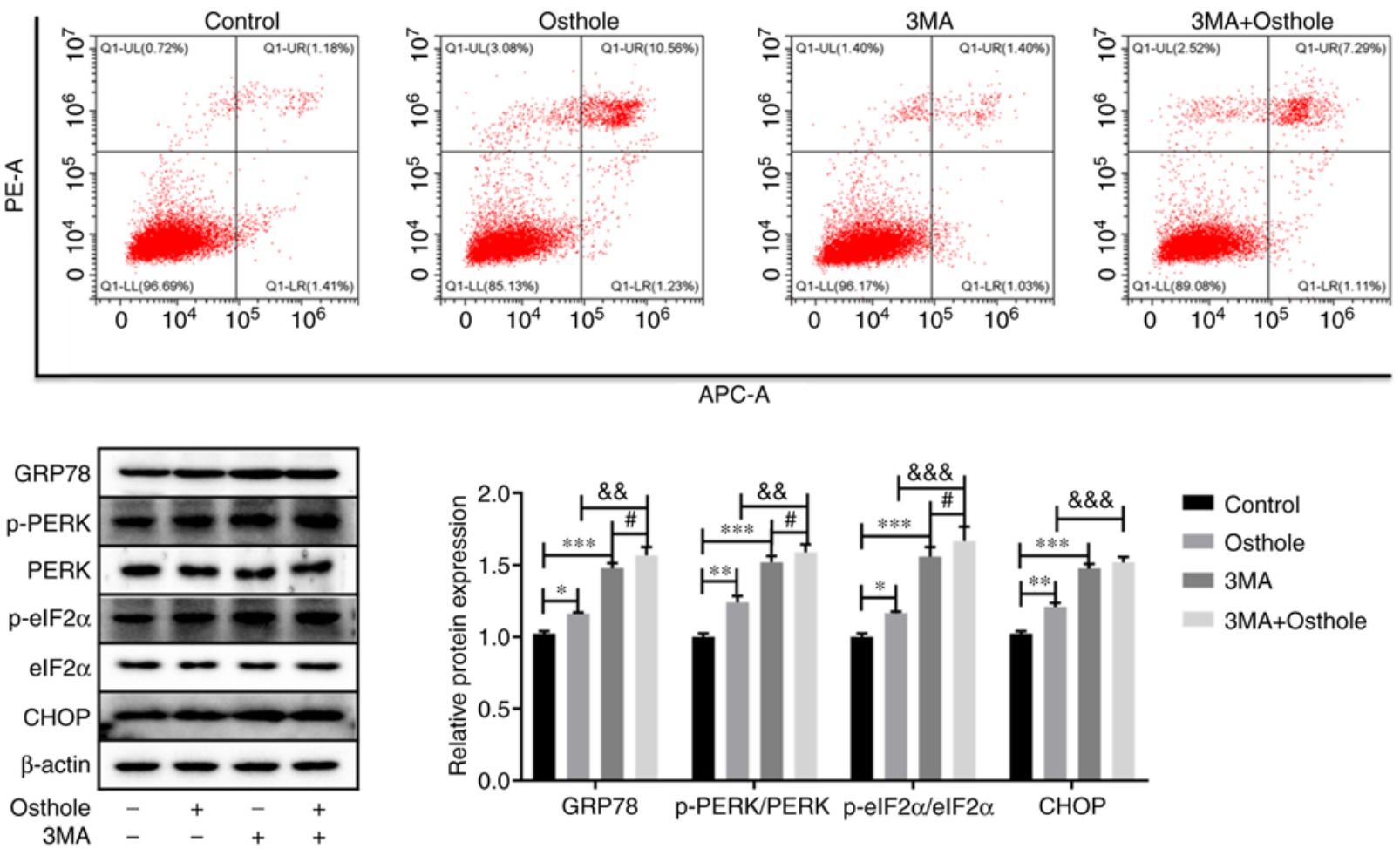

- Control

Osthole

- 3MA

3MA+Osthole

Figure 3. Inhibition of autophagy by 3MA modulates osthole-induced apoptosis and endoplasmic reticulum stress in the HT-29 cell line. (A and B) Cell proliferation was measured using a colony formation assay. (C) Cell viability was measured using a Cell Counting Kit- 8 assay. (D and E) Apoptosis analysis was performed using flow cytometry. (F) The protein expression levels of GRP78, p-PERK/PERK, p-elF2 $\alpha /$ elF2 $\alpha$ and CHOP were determined using western blot analysis, and $\beta$-actin was used as a loading control. ${ }^{*} \mathrm{P}<0.05,{ }^{* *} \mathrm{P}<0.01,{ }^{* * *} \mathrm{P}<0.001$ vs. control group. ${ }^{~} \mathrm{P}<0.05,{ }^{\# \# \#} \mathrm{P}<0.001$ vs. 3MA group. ${ }^{\text {\&\& }} \mathrm{P}<0.01$, \&\&\& $\mathrm{P}<0.001$ vs. osthole group. GRP78, 78 kDa glucose-regulated protein; p-, phosphorylated; PERK, protein kinase R (PKR)-like endoplasmic reticulum kinase; elF2 $\alpha$, eukaryotic initiation factor $2 \alpha$; $3 \mathrm{MA}, 3$-methyladenine.

Furthermore, the apoptosis of the HT-29 cells was examined using flow cytometry and it was found that the apoptosis of the HT-29 cells co-treated with osthole and 3MA was significantly higher compared with that in cells treated with $3 \mathrm{MA}$ alone $(\mathrm{P}<0.01)$, and significantly lower compared with that in the osthole treatment alone group $(\mathrm{P}<0.01$; Fig. 3D and $\mathrm{E})$, indicating that the inhibition of autophagy by $3 \mathrm{MA}$ could attenuate osthole-induced cell apoptosis.
The present study also examined the changes of ERS in the HT-29 cells treated with osthole and 3MA. The results revealed that the protein expression levels of GRP78, p-PERK/PERK, p-elF2 $\alpha /$ elF2 $\alpha$ and CHOP were significantly increased following osthole or 3MA treatment alone, compared with that in the control group $(\mathrm{P}<0.05)$. Furthermore, the HT-29 cells treated with osthole and 3MA exhibited significantly increased protein expression level of GRP78, p-PERK/PERK and p-elF2 $\alpha / \mathrm{elF} 2 \alpha$ 


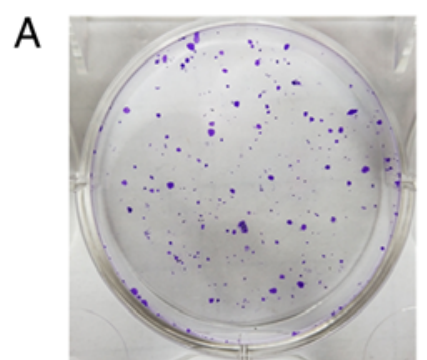

Control

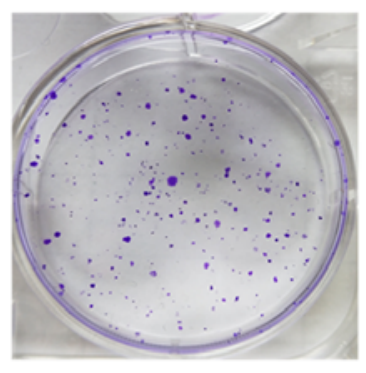

4-PBA

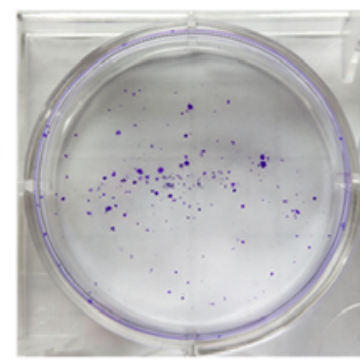

Osthole

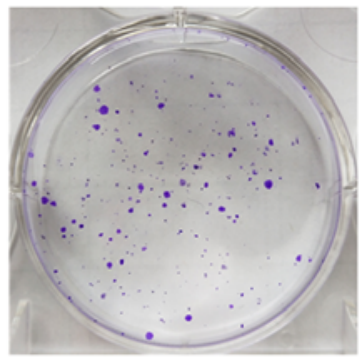

4-PBA+Osthole
B

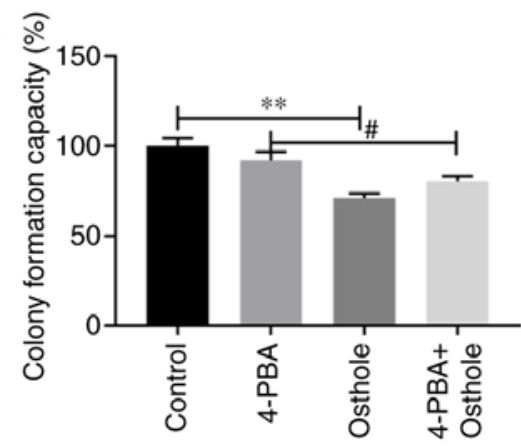

C

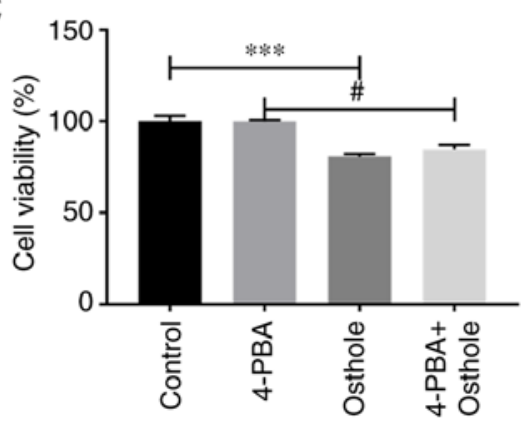

$\mathrm{D}$

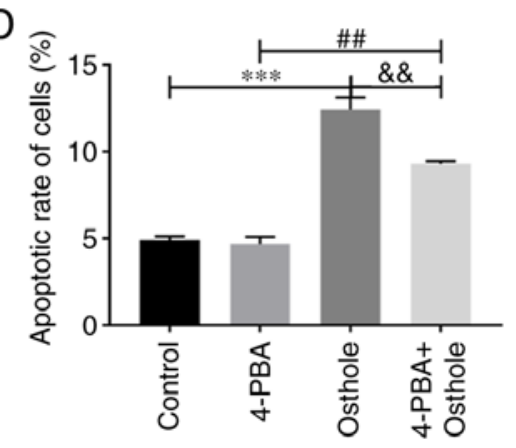

E
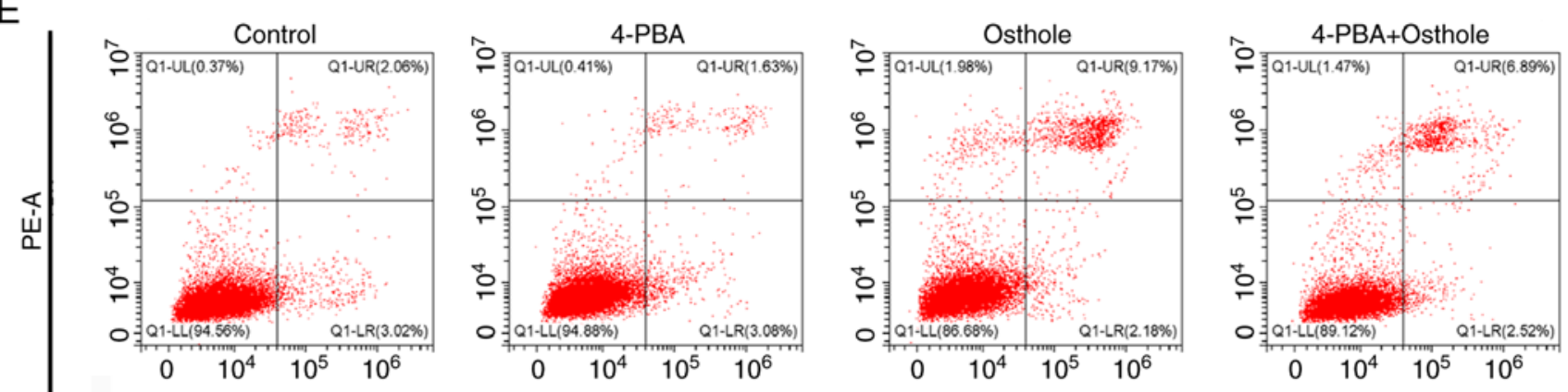

APC-A

F
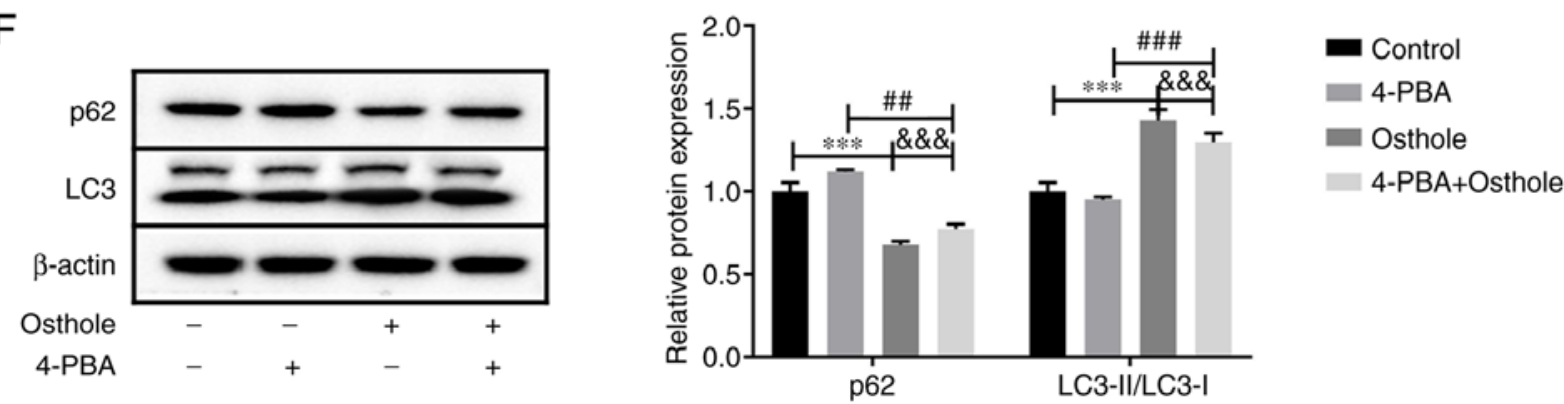

Figure 4. Suppression of endoplasmic reticulum stress with 4-PBA attenuates osthole-induced apoptosis and autophagy in the HT-29 cell line. (A and B) Cell proliferation was measured using a colony formation assay. (C) Cell viability was measured using a Cell Counting Kit-8 assay. (D and E) Apoptosis analysis was performed using flow cytometry. (F) The protein expression levels of p62 and LC3 were determined using western blot analysis, and $\beta$-actin was used as a loading control. ${ }^{* *} \mathrm{P}<0.01,{ }^{* * *} \mathrm{P}<0.001$ vs. control group. ${ }^{\#} \mathrm{P}<0.05,{ }^{\# \#} \mathrm{P}<0.01,{ }^{\# \# \#} \mathrm{P}<0.001$ vs. 4-PBA group. ${ }^{\& \&} \mathrm{P}<0.01,{ }^{\text {\&\&\&}} \mathrm{P}<0.001$ vs. osthole group. LC3, microtubule-associated protein light chain 3; 4-PBA, 4-phenylbutyric acid.

compared with that in the osthole or 3MA alone treatment groups $(\mathrm{P}<0.01)$, while the protein expression level of $\mathrm{CHOP}$ was significantly increased compared with that in the osthole group (Fig. 3E). These results demonstrated that inhibition of autophagy by 3MA may enhance osthole-induced ERS.

Effects of osthole combined with the ERS inhibitor, 4-PBA, on the HT-29 cells. It was observed that inhibition of autophagy reduced apoptosis and enhanced ERS induced by osthole. To further characterize the role of ERS in osthole-induced apoptosis and autophagy, the ERS inhibitor, 4-PBA, was used to treat the HT-29 cells, and colony formation (Fig. 4A and B) and CCK-8 (Fig. 4C) assays were performed to detect cell proliferation and viability, respectively. The results revealed that 4-PBA had no significant effects on cell proliferation and viability compared with that in the control group, and the 

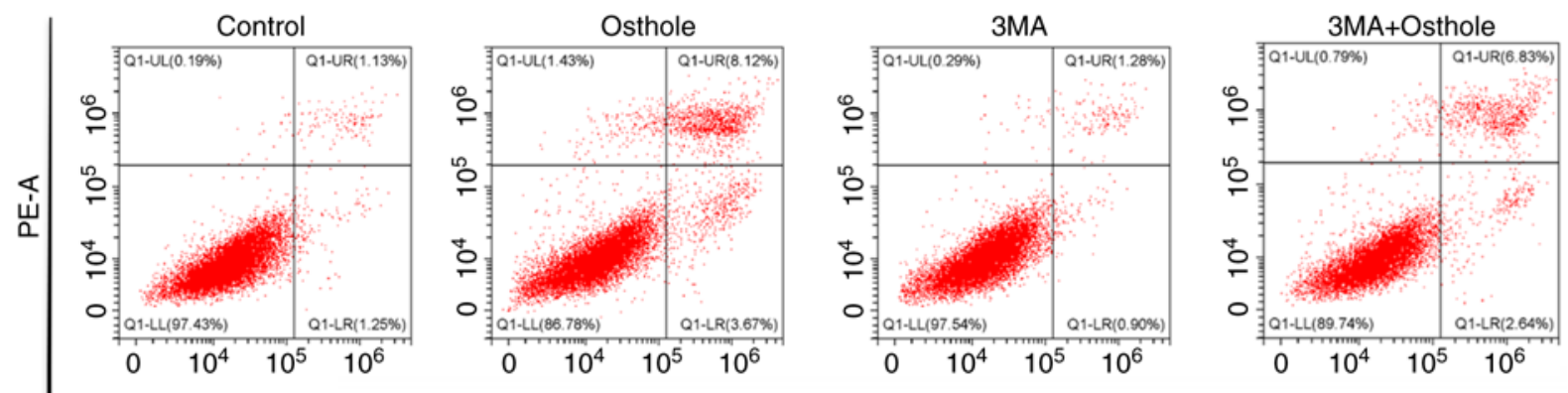

APC-A
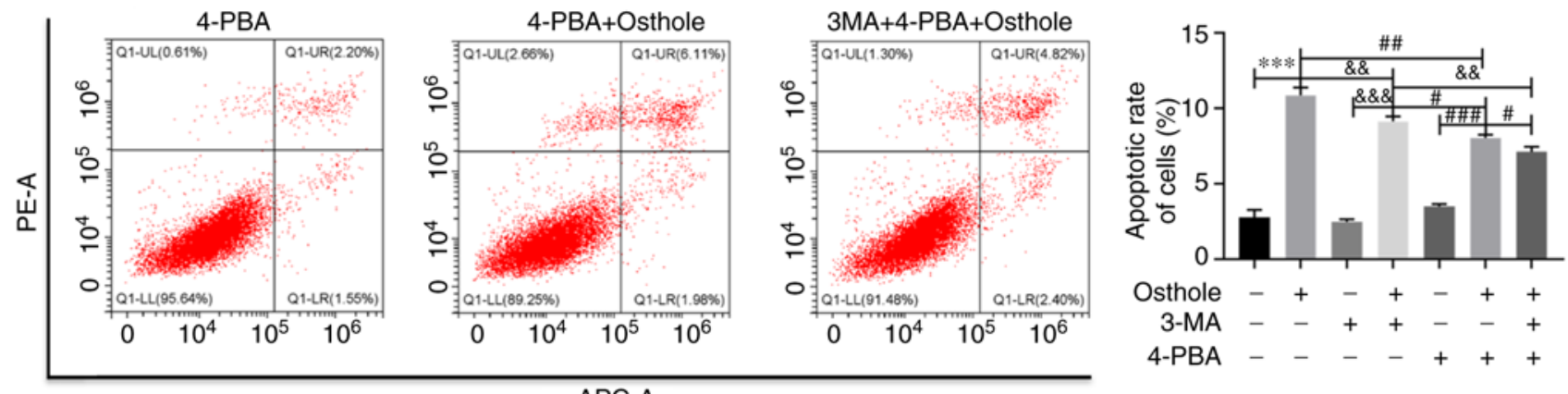

Figure 5. Combined effects of osthole, $3 \mathrm{MA}$ and 4-PBA on apoptosis in the HT-29 cell line. ${ }^{* * * *} \mathrm{P}<0.001$ vs. control group. ${ }^{\& \&} \mathrm{P}<0.01$, \&\&\& $\mathrm{P}<0.001$ vs. $3 \mathrm{MA}+$ osthole group. ${ }^{\#} \mathrm{P}<0.05,{ }^{\# \#} \mathrm{P}<0.01,{ }^{\# \# \#} \mathrm{P}<0.001$ vs. 4-PBA + osthole group. 3MA, 3-methyladenine; 4-PBA, 4-phenylbutyric acid.

co-treatment of 4-PBA and osthole also had no significant effects on cell proliferation and viability compared with that in the osthole group $(\mathrm{P}>0.05)$. However, the proliferation and viability of the HT-29 cells was significantly decreased following osthole and 4-PBA co-treatment compared with that in the 4-PBA group $(\mathrm{P}<0.05)$. In addition, the effects of osthole and 4-PBA co-treatment on the apoptosis of the HT-29 cells was examined using flow cytometry (Fig. 4D and E), and it was found that 4-PBA had no significant effects on apoptosis, whereas cells co-treated with osthole and 4-PBA exhibited significantly higher levels of apoptosis compared with that in the 4-PBA group and lower levels compared with that in the osthole group $(\mathrm{P}<0.01)$. Furthermore, the protein expression levels of autophagy-related proteins were detected using western blot analysis (Fig. 4F). The results demonstrated that $4-\mathrm{PBA}$ had no significant effects on the protein expression levels of p62 and LC3-II/LC3-I $(\mathrm{P}>0.05)$. However, following co-treatment with osthole and 4-PBA, the protein expression level of p62 was significantly lower compared with that in the 4-PBA group and significantly higher compared with that in the osthole group. Furthermore, the protein expression level of LC3-II/LC3-I was significantly increased compared with that in the 4-PBA group and significantly decreased compared with that in the osthole group $(\mathrm{P}<0.01)$, indicating that suppression of ERS with 4-PBA attenuated osthole-induced cell apoptosis and autophagy.

Combined effect of osthole, 3MA and 4-PBA on apoptosis in the HT-29 cells. Since the inhibition of autophagy and suppression of ERS were found to attenuate osthole-induced cell apoptosis separately, the present study further analyzed the combined effects of osthole, 3MA and 4-PBA on the apoptosis of the HT-29 cells using flow cytometry (Fig. 5). It was found that cells co-treated with osthole, 3MA and 4-PBA exhibited significantly lower levels of apoptosis compared with that in the 3MA and osthole or 4-PBA and osthole groups $(\mathrm{P}<0.05)$. These results demonstrated that inhibition of autophagy and ERS, at the same time, significantly attenuated osthole-induced colorectal cancer cell apoptosis. Of note, 4-PBA and osthole treatment reduced apoptosis more significantly compared with that in cells treated with 3MA and osthole $(\mathrm{P}<0.05)$, suggesting that suppression of ERS with 4-PBA played a more important role in alleviating osthole-induced cell apoptosis.

\section{Discussion}

Due to its resistance to anticancer agents, colon cancer has poor outcomes in the clinical setting (2). Therefore, identifying effective drugs for the treatment of colon cancer is crucial. The ability to induce apoptosis has been accepted as a mechanism of action for anticancer drugs, and most of the conventional anticancer drugs are apoptosis inducers, including cisplatin, oxaliplatin and cyclophosphamide (24). Therefore, identifying new agents that induce apoptosis in cancer cells offers potentially useful approaches to improving patient responses to conventional chemotherapy (9).

Accumulating evidence has shown that osthole exerts anticancer effects in different cell lines $(3,8,9)$, yet its preclinical significance and biological role in colon cancer remains unclear. Huang et al (10) found that osthole reduced the viability of the human colon cancer cells, HCT116 and SW480. Consistent with these results, in the present study, osthole inhibited the proliferation and viability of the HT-29 cell line in a dose-dependent manner. Apoptosis occurs via 
2 signaling pathways: The mitochondrial (intrinsic) pathway and the death receptor (extrinsic) pathway. The Bcl-2 protein family regulates the intrinsic pathway by controlling outer mitochondrial membrane integrity (25). Bcl-2 and Bax are important proteins in the Bcl-2 family, which exert anti- and pro-apoptotic effects, respectively. Upregulation of Bax and cleaved caspase-3, and downregulation of Bcl-2 play key roles in inducing cell apoptosis $(26,27)$. Similarly, apoptosis was observed in osthole-treated HT-29 cells in the present study. Furthermore, osthole reduced Bcl-2 and promoted cleaved caspase-3 activation. Therefore, the results of the present study suggested that osthole may induce apoptosis of the HT-29 cells via the intrinsic pathway, which has also been observed in human breast cancer, lung cancer and colon carcinoma cells $(3,9,10)$.

Autophagy, or type II cell death, is an essential cellular process responsible for the degradation of organelles, proteins and other cytoplasmic components such as the cytoplasmic membrane. It fulfils a dual role in all types of cancer, including coloncancer, with both tumor-promoting and tumor-suppressing properties $(28,29)$. LC3 includes 2 forms (LC3-I and LC3-II), in which LC3-II is the autophagosome-associated form, which is converted from the cytosolic form, LC3-I (8). The turnover of LC3-II is often used as a marker of autophagic activity (28). p62, also known as sequestosome 1 , is an adaptor protein that binds to LC3, which is also a substrate for selective autophagy and mitophagy (30). Total p62 protein expression level is negatively associated with autophagic flux (31). The accumulation of p62 is critical for tumorigenesis and p62 has been found to be highly expressed in colon cancer tissues $(32,33)$. In the present study, it was found that the ratio of LC3-II/LC3-I was significantly increased, whereas the protein expression level of p62 was decreased following osthole treatment. Osthole was shown to induce autophagy in human ovarian cancer cells (8). The results of the current study suggested that osthole promoted autophagy and p62 degradation in human colorectal cancer cells.

The UPR is governed by the action of 3 ER sensors: PERK, IRE1 $\alpha$ and ATF6. Under conditions of ERS, GRP78 is released from these 3 sensors (34). Dimerization and subsequent phosphorylation of PERK activates PERK, so it can phosphorylate eIF $2 \alpha$ and induce the translation of ATF4, which consequently enhances the transcription of CHOP, a proapoptotic transcription factor $(18,35,36)$. Osthole has been shown to activate the ERS signaling pathway in normal human hepatocytes and breast cancer cells $(21,37)$. Similarly, the results of the present study demonstrated that the ERS signaling proteins, GRP78, p-PERK/PERK, p-elF2 $\alpha /$ elF $2 \alpha$ and CHOP, were upregulated following osthole treatment in the HT-29 cell line.

Autophagy and the UPR are fundamental mechanisms involved in the regulation of cellular responses to environmental and genetic stresses. Both pathways are interconnected and regulate cellular responses to apoptotic stimuli (38). ERS can induce autophagy via at least 2 UPR pathways, PERK-elF2 $\alpha$ and IRE1 $\alpha$ (17). The compound, 4-PBA, has been used as a selective inhibitor of ERS. In the present study, autophagy was found to be important for cell apoptosis due to pharmacological ERS. Blockade of ERS induction via 4-PBA attenuated osthole-induced apoptosis and autophagy. Consequently, these findings suggested that osthole-induced ERS plays an important role in the crosstalk between apoptosis and autophagy. Osthole could induce apoptosis in colorectal cancer cells, and its effects are partly mediated by the ERS pathway. Autophagy can alleviate ERS by degrading unfolded or aggregated proteins $(17,18)$. The compound, 3MA, is a known inhibitor of autophagy. In the current study, inhibiting cell autophagy with 3MA attenuated osthole-induced cell apoptosis, but enhanced the expression levels of ERS-related proteins following osthole treatment. These results indicated that autophagy played an anti-tumorigenic role in apoptosis, which was induced by osthole. Furthermore, the effects of autophagy inhibitors on alleviating osthole-induced apoptosis was less prominent compared with that in cells treated with ERS inhibitors, suggesting that the activation of autophagy may be dependent on ERS during this process.

In conclusion, osthole inhibited the proliferation and viability, and induced apoptosis of the HT-29 cell line via activation of autophagy and the ERS pathway, and ERS plays an important role in osthole-induced cell apoptosis. Thus, osthole may be a promising candidate for the treatment of human colon cancer.

\section{Acknowledgements}

Not applicable.

\section{Funding}

The present study was supported by the National Natural Science Foundation of China (grant no. 81873073) and Science and Technology Development Fund of Hospital of Chengdu University of Traditional Chinese Medicine (grant no. 18MZ27).

\section{Availability of data and materials}

The datasets used and/or analyzed during the current study are available from the corresponding author on reasonable request.

\section{Authors' contributions}

YC conceived and designed the research. XHZ performed most of the experiments. JK and ZDZ performed parts of the experiments. XHZ, JK and ZDZ analyzed the data. XHZ and YC wrote the manuscript. All authors read and approved the final manuscript. YC and XHZ confirm the authenticity of all the raw data.

\section{Ethics approval and consent to participate}

Not applicable.

\section{Patient consent for publication}

Not applicable.

\section{Competing interests}

The authors declare that they have no competing interests. 


\section{References}

1. Bray F, Ferlay J, Soerjomataram I, Siegel RL, Torre LA and Jemal A: Global cancer statistics 2018: GLOBOCAN estimates of incidence and mortality worldwide for 36 cancers in 185 countries. CA Cancer J Clin 68: 394-424, 2018.

2. $\mathrm{Hu} \mathrm{T}, \mathrm{Li} \mathrm{Z}$, Gao CY and Cho CH: Mechanisms of drug resistance in colon cancer and its therapeutic strategies. World J Gastroenterol 22: 6876-6889, 2016.

3. Wang L, Peng Y, Shi K, Wang H, Lu J, Li Y and Ma C: Osthole inhibits proliferation of human breast cancer cells by inducing cell cycle arrest and apoptosis. J Biomed Res 29: 132-138, 2015.

4. Tang DZ, Hou W, Zhou Q, Zhang M, Holz J, Sheu TJ, Li TF, Cheng SD, Shi Q, Harris SE, et al: Osthole stimulates osteoblast differentiation and bone formation by activation of beta-catenin-BMP signaling. J Bone Miner Res 25: 1234-1245, 2010.

5. Bao Y, Meng X, Liu F, Wang F, Yang J, Wang H and Xie G: Protective effects of osthole against inflammation induced by lipopolysaccharide in BV2 cells. Mol Med Rep 17: 4561-4566, 2018.

6. Li Y, Li Y, Shi F, Wang L, Li L and Yang D: Osthole attenuates right ventricular remodeling via decreased myocardial apoptosis and inflammation in monocrotaline-induced rats. Eur J Pharmacol 818: 525-533, 2018

7. Liu WB, Zhou J, Qu Y, Li X, Lu CT, Xie KL, Sun XL and Fei Z: Neuroprotective effect of osthole on MPP+-induced cytotoxicity in PC12 cells via inhibition of mitochondrial dysfunction and ROS production. Neurochem Int 57: 206-215, 2010.

8. Liang J, Zhou J, Xu Y, Huang X, Wang X, Huang W and Li H: Osthole inhibits ovarian carcinoma cells through LC3-mediated autophagy and GSDME-dependent pyroptosis except for apoptosis. Eur J Pharmacol 874: 172990, 2020.

9. Xu XM, Zhang ML, Zhang Y and Zhao L: Osthole induces lung cancer cell apoptosis through inhibition of inhibitor of apoptosis family proteins. Oncol Lett 12: 3779-3784, 2016.

10. Huang SM, Tsai CF, Chen DR, Wang MY and Yeh WL: p53 is a key regulator for osthole-triggered cancer pathogenesis. Biomed Res Int 2014: 175247, 2014

11. Jarząb A, Grabarska A, Skalicka-Woźniak K and Stepulak A: Pharmacological features of osthole. Postepy Hig Med Dosw (Online) 71: 411-421, 2017.

12. D'Arcy MS: Cell death: A review of the major forms of apoptosis, necrosis and autophagy. Cell Biol Int 43: 582-592, 2019.

13. Oakes S: Endoplasmic reticulum stress signaling in cancer cells Am J Pathol 190: 934-946, 2020.

14. Guzel E, Arlier S, Guzeloglu-Kayisli O, Tabak MS, Ekiz T, Semerci N, Larsen K, Schatz F, Lockwood CJ and Kayisli UA: Endoplasmic reticulum stress and homeostasis in reproductive physiology and pathology. Int J Mol Sci 18: 792, 2017.

15. Almanza A, Carlesso A, Chintha C, Creedican S, Doultsinos D, Leuzzi B, Luís A, McCarthy N, Montibeller L, More S, et al: Endoplasmic reticulum stress signalling-from basic mechanisms to clinical applications. FEBS J 286: 241-278, 2019.

16. Karagöz GE, Acosta-Alvear D and Walter P: The unfolded protein response: Detecting and responding to fluctuations in the protein-folding capacity of the endoplasmic Reticulum. Cold Spring Harbor Perspect Biol 11: a033886, 2019.

17. Sano R and Reed JC: ER stress-induced cell death mechanisms. Biochim Biophys Acta 1833: 3460-3470, 2013.

18. Verfaillie T, Salazar M, Velasco G and Agostinis P: Linking ER Stress to autophagy: Potential implications for cancer therapy. Int J Cell Biol 2010: 930509, 2010.

19. Latorraca LB, Feitosa WB, Mariano C, Moura MT, Fontes PK, Nogueira MFG and Paula-Lopes FF: Autophagy is a pro-survival adaptive response to heat shock in bovine cumulus-oocyte complexes. Sci Rep 10: 13711, 2020.
20. Di S, Fan C, Ma Z, Li M, Guo K, Han D, Li X, Mu D and Yan X: PERK/eIF-2 $\alpha /$ CHOP pathway dependent ROS generation mediates butein-induced non-small-cell lung cancer apoptosis and G2/M phase arrest. Int J Biol Sci 15: 1637-1653, 2019.

21. Park W, Park S, Song G and Lim W: Inhibitory effects of osthole on human breast cancer cell progression via induction of cell cycle arrest, mitochondrial dysfunction, and ER stress. Nutrients 11: 2777, 2019.

22. Maiuri MC, Zalckvar E, Kimchi A and Kroemer G: Self-eating and self-killing: Crosstalk between autophagy and apoptosis. Nat Rev Mol Cell Biol 8: 741-752, 2007.

23. Song S, Tan J, Miao Y, Li M and Zhang Q: Crosstalk of autophagy and apoptosis: Involvement of the dual role of autophagy under ER stress. J Cell Physiol 232: 2977-2984, 2017.

24. Pistritto G, Trisciuoglio D, Ceci C, Garufi A and D'Orazi G: Apoptosis as anticancer mechanism: Function and dysfunction of its modulators and targeted therapeutic strategies. Aging 8: 603-619, 2016.

25. Chipuk JE, Moldoveanu T, Llambi F, Parsons MJ and Green DR: The BCL-2 family reunion. Mol Cell 37: 299-310, 2010.

26. Shokoohinia Y, Jafari F, Mohammadi Z, Bazvandi L, Hosseinzadeh L, Chow N, Bhattacharyya P, Farzaei MH, Farooqi AA, Nabavi SM, et al: Potential anticancer properties of osthol: A comprehensive mechanistic review. Nutrients 10: 36 , 2018.

27. Siddiqui WA, Ahad A and Ahsan H: The mystery of BCL2 family: Bcl-2 proteins and apoptosis: An update. Arch Toxicol 89: 289-317, 2015.

28. Devenport SN and Shah YM: Functions and implications of autophagy in colon cancer. Cells 8: 1349, 2019.

29. Mathew R, Karantza-Wadsworth V and White E: Role of autophagy in cancer. Nat Rev Cancer 7: 961-967, 2007.

30. Mizushima N and Komatsu M: Autophagy: Renovation of cells and tissues. Cell 147: 728-741, 2011.

31. Zhang Y, Ren S, Liu Y, Gao K, Liu Z and Zhang Z: Inhibition of starvation-triggered endoplasmic reticulum stress, autophagy, and apoptosis in ARPE-19 cells by taurine through modulating the expression of calpain-1 and calpain-2. Int J Mol Sci 18: 2146, 2017.

32. Lei C, Zhao B, Liu L, Zeng X, Yu Z and Wang X: Expression and clinical significance of p62 protein in colon cancer. Medicine (Baltimore) 99: e18791, 2020

33. Zhang H, Zhang Y, Zhu X, Chen C, Zhang C, Xia Y, Zhao Y, Andrisani $\mathrm{O}$ and Kong L: DEAD box protein 5 inhibits liver tumorigenesis by stimulating autophagy via interaction with p62/SQSTM1. Hepatology 69: 1046-1063, 2019.

34. Gong J, Wang XZ, Wang T, Chen JJ, Xie XY, Hu H, Yu F, Liu HL, Jiang XY and Fan HD: Molecular signal networks and regulating mechanisms of the unfolded protein response. J Zhejiang Univ Sci B 18: 1-14, 2017

35. Sisinni L, Pietrafesa M, Lepore S, Maddalena F, Condelli V, Esposito $\mathrm{F}$ and Landriscina M: Endoplasmic reticulum stress and unfolded protein response in breast cancer: The balance between apoptosis and autophagy and its role in drug resistance. Int J Mol Sci 20: 857, 2019.

36. Woo KJ, Lee TJ, Lee SH, Lee JM, Seo JH, Jeong YJ, Park JW and Kwon TK: Elevated gadd153/chop expression during resveratrol-induced apoptosis in human colon cancer cells. Biochem Pharmacol 73: 68-76, 2007.

37. Shen $\mathrm{Z}$, Chen $\mathrm{J}$ and $\mathrm{Lu} \mathrm{H}$ : Osthole induced apoptosis in human normal liver cells by regulating cell proliferation and endoplasmic reticulum stress. Environ Toxicol 34: 768-776, 2019.

38. Mokarram P, Albokashy M, Zarghooni M, Moosavi MA, Sepehri Z, Chen QM, Hudecki A, Sargazi A, Alizadeh J, Moghadam AR, et al: New frontiers in the treatment of colorectal cancer: Autophagy and the unfolded protein response as promising targets. Autophagy 13: 781-819, 2017.

This work is licensed under a Creative Commons Attribution-NonCommercial-NoDerivatives 4.0 International (CC BY-NC-ND 4.0) License. 